\title{
Die nackte weibliche Brust als Sittlichkeits- und Rechtsproblem
}

Anja Schmidt

2021-07-19T13:07:16

Ende Juni löste eine in Berlin lebende Frau einen Polizeieinsatz aus, weil sie bei hochsommerlichen Temperaturen am Wasserspielplatz „Plansche“ im Plänterwald mit freiem Oberkörper ruhte und ihre Brust auf Aufforderung der Parkaufseher hin nicht bedecken wollte. Das Argument, sie wolle mit Männern mit freiem Oberköper gleichbehandelt werden, wurde nicht akzeptiert. Ihre unbedeckte Brust sei störend, zudem „gibt es hier Kinder“. Letztlich fühlte sie sich gezwungen (mit ihrem Kind) zu gehen und rief die Initiative "Gleiche Brust für alle" ins Leben. Im Rahmen dieser Bewegung nahmen am 10. Juli an einer Oben-Ohne-Fahrrad-Demo unter dem Motto „No Nipple Is Free Until All Nipples Are Free“ hunderte Frauen und Männer mit freiem Oberköper teil, um gegen die Sexualisierung (und die damit verbundenen Tabuisierung) der weiblichen Brust zu demonstrieren.

Ist ein Polizeieinsatz wegen einer unbedeckten weiblichen Brust an einem öffentlich zugänglichen Wasserspielplatz mit Liegewiese im Park eine staatlich zu verantwortende gleichheitswidrige Sexualisierung der weiblichen Brust?

\section{Mögliche rechtliche Grundlagen für das polizeiliche Einschreiten}

Die Nutzungsordnung des von der Berliner Verwaltung betriebenen Wasserspielplatzes bildet keine rechtliche Grundlage für das polizeiliche Eingreifen. Aus ihr ergibt sich lediglich, dass „von allen Gästen Straßen- oder Alltagskleidung bzw. handelsübliche Badekleidung, wie z.B. Badehose, Badeshorts, Bikini, Badeanzug, Burkini zu tragen“ ist. Die Nutzerin hatte eine Badehose getragen, was den Vorgaben entspricht. Denn durch die Nutzungsordnung war der Ort einem Textilstrand ähnlich gewidmet und es ist nicht unüblich, dass Frauen sich an Textilstränden „oben ohne“ aufhalten. Eine engere Auslegung der Nutzungsordnung würde tatsächlich die Frage aufwerfen, warum die weibliche Brust im Gegensatz zur männlichen Brust bedeckt werden muss. Ein sachlich nachvollziehbarer Grund dafür ist nicht ersichtlich - eine weibliche Brust ist nicht von sich aus anstößig oder sexuell, insbesondere nicht, wenn sie in einer strandartigen Situation sichtbar ist.

Das Vorgehen der Polizei ließe sich nur aufgrund der polizeilichen Generalklausel rechtfertigen, genauer dann, wenn die nackte weibliche Brust ein öffentliches Ärgernis im Sinne des $\S 183 a$ StGB erregt oder sich die Allgemeinheit im Sinne des $\S 118$ OWiG belästigt. Die Erregung eines öffentlichen Ärgernisses nach § 183a StGB setzt voraus, dass öffentlich eine sexuelle Handlung vorgenommen wird. Das Entblößen der Brust, auch der weiblichen Brust, an einem Wasserspielplatz mit Liegewiese im Hochsommer lässt sich schwerlich als sexuelle Handlung 
einordnen. Denn hier steht schon dem äußeren Erscheinungsbild nach der Wunsch im Vordergrund, sich bei hohen Temperaturen mit möglichst wenig Kleidung am Körper zu entspannen. Es kommt nicht darauf an, dass dies andere als störend empfinden mögen.

Schwieriger ist die Beurteilung, ob eine Belästigung der Allgemeinheit nach § 118 OWiG vorliegt. Denn diese Norm setzt mit sehr unscharfen Begriffen voraus, dass eine "grob ungehörige Handlung" vorgenommen wird, „die geeignet ist, die Allgemeinheit zu belästigen oder zu gefährden und die öffentliche Ordnung zu beeinträchtigen“. Was ist "grob ungehörig“? Was kann die Allgemeinheit belästigen oder gefährden oder die öffentliche Ordnung beeinträchtigen? Und: woran wird dies bemessen? Am Gefühl der Belästigung oder Störung zufällig Anwesender, der Parkaufseher oder der Polizei? An der Messlatte allgemeinen Sittlichkeitsempfindens oder anhand rechtlicher Maßstäbe?

\section{Schutz individueller Rechte statt des Schutzes von Sittlichkeit}

Als grob ungehörig definiert Lothar Senge in der aktuellen 5. Auflage des Karlsruher Kommentars zu § 118 OWiG mit Bezug auf ältere Rechtsprechung ein Verhalten, das sich „bewusst nicht in die für das gedeihliche Zusammenleben der jeweiligen Rechtsgemeinschaft erforderliche Ordnung einfügt und dadurch im deutlichen Widerspruch zur Gemeinschaftsordnung steht". Später verwendet er die Begriffe der "anerkannten Regeln von Sitte, Anstand und Ordnung“ anstelle des Begriffs der Gemeinschaftsordnung. Belästigend sei eine Handlung, wenn sie Dritten ein nicht nur geringfügiges Unbehagen zufügt. § $118 \mathrm{OWiG}$ ist damit ersichtlich eine Norm, die in der Tradition eines Sittlichkeitsrechts steht, das gesellschaftlich allgemein übliche Umgangsweisen schützt. Es geht also gerade nicht um den Schutz von Rechten, sondern um den Schutz des gesellschaftlich Üblichen oder allgemein moralisch Anerkannten, um zumindest ein nicht nur geringfügiges Unbehagen zu vermeiden.

In einem normativen Sinne ist es jedoch nicht Aufgabe des Rechts, herrschende Moralauffassungen zu schützen. Insbesondere im Bereich von Nacktheit und Sexualität ist dies aufgrund der Pluralisierung moralischer Vorstellungen auch gar nicht mehr möglich. Aufgabe des Rechts ist es vielmehr, Freiheit unter anderem durch individuelle Freiheitsrechte zu gewährleisten. Damit sind auch individuelle moralische Entscheidungen und deren Umsetzung geschützt, soweit hierdurch die individuellen Rechte anderer nicht verletzt werden. Auf diese Weise schützt das Recht unter anderem vor faktisch herrschenden sozialmoralischen Anschauungen. Beispielsweise stehen homosexuellen Menschen die gleichen Rechte wie heterosexuellen Menschen zu, auch wenn es Menschen gibt, die Homosexualität als anstößig oder moralisch verwerflich empfinden. Vor diesem Hintergrund bedarf es einer restriktiven, auf individuelle Rechte bezogenen Auslegung des $\S 118 \mathrm{OWiG}$. In einem Beschluss des OLG Hamburg vom 2.6.2005 (Az.: III-28/05 3 Ss 20/05 OWi, zum öffentlichen Ausstellen plastinierter menschlicher Leichen) wurden deshalb die unbestimmten Rechtsbegriffe des $§ 118$ OWiG mit einem Rückgriff auf die 
grundgesetzliche Werteordnung konkretisiert, genauer wurde auf eine Missachtung der von Art. 1 Abs. 1 GG geschützten Menschenwürde abgestellt und die Meinungsund Kunstfreiheit nach Art. 5 Abs. 1 und 3 GG einbezogen.

\section{Anstand, Schamgefühl und Nacktheit}

Im Zusammenhang mit Nacktheit geht die Rechtsprechung in jüngerer Zeit davon aus, dass $\S 118$ OWiG einschlägig ist, wenn „das Scham- und Anstandsgefühl der ungewollt mit fremder Nacktheit konfrontierten Menschen nachhaltig tangiert wird“ (VGH Mannheim, Beschluss v. 3.9.2002, Az.: 1 S 972/02) oder „wenn die Handlung in einem so deutlichen Widerspruch zur Gemeinschaftsordnung steht, dass sie jeder billig und gerecht denkende Bürger als grobe Rücksichtslosigkeit gegenüber jedem Mitbürger ansehen würde, sie sich also gleichsam als eine Missachtung der durch die Gemeinschaftsordnung geschützten Interessen darstellt" (OLG Karlsruhe, Beschluss v. 4.5.2000, Az.: 2 Ss 166/99; VG Karlsruhe, Beschluss v. 2.6.2005, Az.: 6 K 1058/05). Deshalb werden das Nacktradeln oder Nacktjoggen im städtischen Raum, bei dem der „Schambereich“, oft ein Penis, sichtbar ist, regelmäßig aufgrund $\S 118 \mathrm{OWiG}$ in Verbindung mit der polizeilichen Generalklausel verboten. Teils wird auch, unabhängig vom Geschlecht, auf die Nacktheit insgesamt abgestellt. Letztlich kommt es auf die Umstände des Einzelfalles, konkret darauf an, ob an dem fraglichen Ort mit Nacktheit zu rechnen ist. Das nackte Baden an Stränden oder in Schwimmbädern wird dabei als nicht mehr unüblich betrachtet, Nacktheit an Orten, wo sie üblicherweise nicht erwartet werden kann, gilt hingegen als „Verletzung des natürlichen nicht übertriebenen Schamgefühls der betroffenen Allgemeinheit" (OLG Karlsruhe, Beschluss v. 4.5.2000, Az.: 2 Ss 166/99; VG Karlsruhe, Beschluss v. 2.6.2005, Az.: 6 K 1058/05).

„Die Allgemeinheit“" als solche kann kein Schamgefühl haben. Dennoch lässt sich anhand dieser Maßstäbe für die nackte weiblichen Brust an der Plansche feststellen, dass es nicht unüblich ist, an einem sommerlichen Wasserspielplatz mit Liegewiese nackte Oberkörper (also gerade nicht einen Penis oder eine Vulva) zu sehen. Das gilt nicht nur für die männliche, sondern auch für die weibliche Brust. Zwar ist es gesellschaftlich üblich, die weibliche Brust stärker zu sexualisieren als die männliche, aber es lässt sich nicht sagen, dass der Anblick einer nackten weiblichem Brust an einem Wasserspielplatz mit Liegewiese so deutlich im Widerspruch zur Gemeinschaftsordnung steht, dass sie jeder billig und gerecht denkende Mensch als grob rücksichtslos betrachten würde. Es lässt sich zwar kaum anhand allgemeiner Merkmale bestimmen lässt, was diesen billig und gerecht denkenden Menschen als Maßstabsfigur ausmacht, dennoch liegt keine grobe, allgemein konsentierte Rücksichtslosigkeit vor, die eine geradezu eine Missachtung der Interessen der Anwesenden darstellen würde. Die Frage nach einer Ungleichbehandlung im Vergleich zu Menschen mit männlicher Brust stellt sich insofern noch gar nicht. Allerdings wäre eine solche Ungleichbehandlung nicht legitim, denn sie würde auf einer Sexualisierung und Tabuisierung der weiblichen Brust beruhen, die jedenfalls an einer strandartigen Badestelle unangemessen ist. 


\section{Grundrechtliche Einordnung}

Eine stärker an der grundrechtlichen Werteordnung orientierte Argumentation kommt zu dem gleichen Ergebnis: Freiheitsrechtlich gedacht geht es beim Zeigen der nackten Brust an einer sommerlichen Wasserplanschstelle um die allgemeine Handlungsfreiheit nach Art. 2 Abs. 1 GG in zweierlei Hinsicht - positiv als die Freiheit, sich nach eigenem Gutdünken in der Öffentlichkeit bewegen zu können, und negativ als die Freiheit, nicht mit Zumutungen, die die Schwelle des rechtlich Hinzunehmenden überschreiten, konfrontiert zu werden. Die abzuwägenden Rechtspositionen verbürgen zudem nach Art. 3 GG gleiche Freiheiten, es darf also nicht diskriminiert werden, insbesondere nicht wegen des Geschlechts (Art. 3 Abs. 2 und 3 S. 1 GG).

Grundsätzlich ist die Freiheit, sich an einer Wasserplanschstelle mit strandartiger Liegewiese mit nacktem Oberkörper aufzuhalten, von der allgemeinen Handlungsfreiheit nach Art. 2 Abs. 1 GG erfasst. Zwar kann eine Nutzungsordnung hier grundsätzlich Einschränkungen vorsehen, allerdings schloss die Nutzungsordnung mit dem Verweis auf die Notwendigkeit von Badebekleidung hier nur vollständige Nacktheit aus. Mit dieser Freiheit ist das Recht abzuwägen, nicht ungewollt mit unzumutbaren Verhaltensweisen anderer konfrontiert zu werden. Ein notwendiger Konfrontationsschutz ist dabei sorgfältig zu begründen, denn nicht jede Lästigkeit sollte zu Einschränkungen der Rechte anderer führen können. Zum einen verletzt eine bloße Lästigkeit gerade keine Rechte, zum anderen ist das, was als lästig empfunden wird, häufig subjektiv. Vorliegend war an der strandartigen Liegewiese am Wasserspielplatz mit nackten Oberkörpern zu rechnen, der Anblick war also nicht unüblich oder unerwartet. Eine Unzumutbarkeit ergibt sich auch nicht daraus, dass die Frau den anderen Nutzer:innen der Wasserplansche ein sexuelles Geschehen aufdrängt hätte. Denn eine nackte weibliche Brust ist zunächst einmal eine nackte weibliche Brust und keine sexuelle Intervention, insbesondere wenn sie auf einer strandartigen Liegewiese zu sehen ist, wo sich viele Menschen in Badekleidung aufhalten. Eine gegenteilige rechtliche Wertung würde die weibliche Brust gegenüber der männlichen Brust gleichheitswidrig sexualisieren. Ein anderes Ergebnis lässt sich auch nicht damit begründen, dass Kinder eine nackte weibliche Brust sehen können. Für Kinder dürften nackte Körper und Körperteile unabhängig von der Sexualisierung durch andere häufig nichts weiter sein als nackte Körper und Körperteile; die weibliche Brust assoziieren sie wohl eher mit dem Stillen. In dem Verweis der Parkaufseher auf die anwesenden Kinder drücken sich vermutlich vor allem das Unbehagen und die Anstandsvorstellungen von Erwachsenen aus.

\section{Keine Sexualisierung weiblicher Körper durch Recht}

Der polizeiliche Platzverweis der Frau mit der unbekleideten Brust von dem Wasserspielplatz stellt sich damit als rechtlich unzulässige und gleichheitswidrige Sexualisierung ihres Körpers und als rechtswidrig dar. Einem rechtlichen Vorgehen dagegen ist Erfolg zu wünschen, auch im Sinne aller Menschen mit weiblich gelesenen Körpern. 
(c)) BY-SA

- 5 - 